\section{Analysis of the Detection Limit on a Micro- electronic Array}

BioTechniques 33:494-496 (September 2002)

The last few years have seen acceleration in the use of microarrays in biological research. More than 200 companies are now engaged in or developing microarray technology (1). DNA microarrays were originally developed to process the huge amount of information provided by genome projects. Because the main benefit of microarrays derives from the ability to analyze thousands of analytes simultaneously, microarray analysis emerged as the preferred technology for gene expression studies. Other major applications include the examination of DNA mutations with a clear focus on single nucleotide polymorphism (6).

In addition, although not routinely used for such applications, microarray technology may have potential in diagnostics and mass screening of a large number of samples (e.g., use of microarray analysis could revolutionize blood testing) (5). In principal, a single chip can combine different microbiological, blood typing, or platelet tests (5). Although the normal applications of microarrays are well established, some issues must be resolved before they can be used routinely for such mass screening.

One issue of particular importance for the use of microarrays in diagnostics is the sensitivity of a specific technology. Microarrays are widely believed to be extremely sensitive, but a critical review of publications reveals that little attention has been paid to the question of sensitivity on an experimental level $(1,5)$. Although specific experimental microarray technology can directly detect $16 \mathrm{~S}$ rRNA in soil extracts, suggesting that it is possible to apply microarrays under certain circumstances for the direct detection of microorganisms without using PCR (7), one report states that the sensitivity of microarrays is slightly inferior to that of Northern blot analyses (8). Therefore, a simple method of testing whether the detection of nucleic acids is possible without amplification would be advantageous.

The sensitivity of an array depends on a large number of factors: the sequence of the capture probes, the method of attachment to the support (10), and the hybridization conditions after the addition of the samples.

A conventional, passive microarray designed for the analysis of many analytes with one sample is unsuitable for screening large numbers of different samples. On a passive microarray, the hybridization of the DNA is dependent on diffusion and not supported by any manipulation such as electronic movement of charged biological molecules (2). Therefore, for our experiments, we chose a microelectronic array technology (4) that allows specific, consecutive attaching of different molecules to a test site. The highest possible sensitivity of this technology (NanoChip ${ }^{\circledR}$ Molecular Biology Workstation; Nanogen, San Diego, CA, USA) was unknown.

We developed a simple method for analyzing the detection limit, ruling out as many of the factors as possible that could influence sensitivity. An oligonucleotide sequence with Cy5 conjugated at the $5^{\prime}$-end and biotin conjugated at the $3^{\prime}$-end, (5'-Cy5-GATCCAAGA-Biotin$\left.3^{\prime}\right)$ was chosen at random and purchased from MWG-Biotech (Ebersberg, Germany). A $100-\mu \mathrm{M}$ stock solution of the oligonucleotide was serially diluted in $50 \mathrm{mM}$ L-histidine buffer using glass distilled water with a conductivity of less than 5 $\mu \mathrm{S} / \mathrm{cm}$. The overall conductivity of the oligonucleotide dilutions was always below $100 \mu \mathrm{S} / \mathrm{cm}$.

We pipetted $60 \mu \mathrm{L}$ of the respective dilutions, as well as $50 \mathrm{mM}$ L-histidine alone (negative control) into a 96-well plate (Nalge Nunc International, Roskilde, Denmark). The NanoChip cartridge and the 96-well plate were placed together into the loader according to the supplier's instructions. To gain the highest possible sensitivity, each sample was attached for $10 \mathrm{~min}$ (the longest possible addressing time), with a current of $400 \mathrm{nA} \pm 20 \%$, to distinct pads on the NanoChip cartridge. The cartridge was scanned in the Molecular Biology Workstation reader at a high gain setting with an accumulation time of $2000 \mu \mathrm{s}$.

The oligonucleotide was from two different lots, and the experiment was repeated three times for each lot. In every experiment, the detection limit was at a concentration of approximately 0.25 pM (Figure 1). Because $20 \mu \mathrm{L}$ were loaded onto the chip, we calculated that this corresponds to approximately $3 \times 10^{6}$ molecules in contact with the chip. In comparison, the detection limit of a 100-bp DNA fragment on a gel stained with ethidium bromide is about $10^{11}$ molecules.

Knowledge of the sensitivity of a given microarray technology makes it easier to calculate the feasibility of using that technology for a certain application. For example, there is only a minor chance of directly detecting (without amplification) pathogenic viruses in plasma such as hepatitis $\mathrm{C}$ virus (HCV) and human immunodeficiency virus (HIV), which are typically present in body fluid in copy numbers of up to $10 \% / \mathrm{mL}$ (data not shown). However, a microelectronic array approach for the

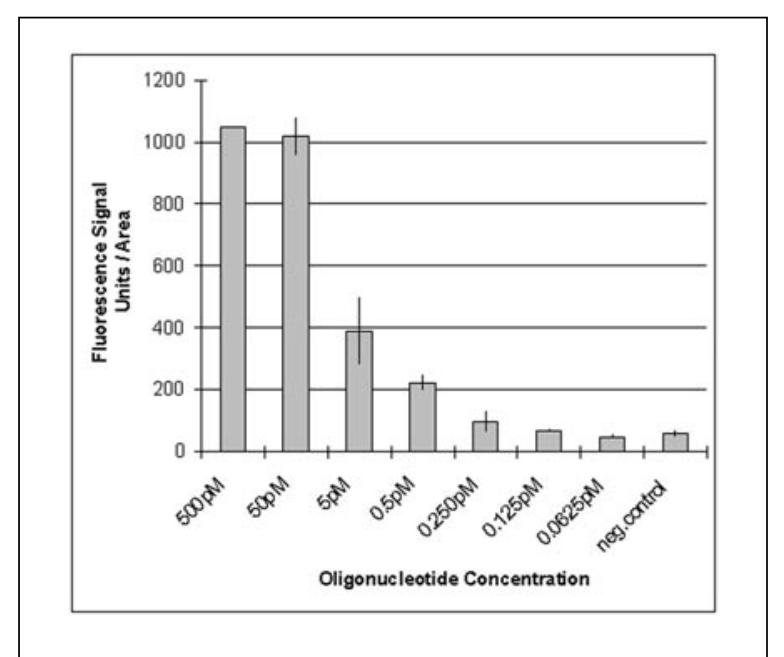

Figure 1. Detection limit of the microelectronic array. An oligonucleotide containing both binding and detection molecules was serially diluted and directly loaded onto the chip. Two pads were addressed for each dilution. The respective concentrations of the dilutions are indicated in the figure. Six cartridges were tested with similar results, showing the reproducibility of the NanoChip microelectronic array technology. 
detection of small numbers of nucleic acids would be possible when amplification and detection are carried out simultaneously on the cartridge (9). Detection methods such as nanoparticles (3) might be another way to improve the sensitivity and eliminate the need for amplification. Our principle for analysis of the detection limit can easily be adopted for any other microarray system and could be a first step for the identification of suitable technologies for potential applications in routine screening of samples without amplification.

\section{REFERENCES}

1.Blohm, D.H. and A. Guiseppi-Elie. 2001. New developments in microarray technology. Curr. Opin. Biotechnol. 12:41-47.

2.Edman, C.F., D.E. Raymond, D.J. Wu, E. Tu, R.G. Sosnowski, W.F. Butler, M. Nerenberg, and M.J.Heller. 1997. Electric field directed nucleic acid hybridization on microchips. Nucleic. Acids Res. 25:4907-4914.

3.Härmä, H., T. Soukka, and T. Lövgren. 2001. Europium nanoparticles and time-resolved fluorescence for ultrasensitive detection of prostate-specific antigen. Clin. Chem. 47:561-568.

4.Heller, M.J., A.H. Forster, and E. Tu. 2000. Active microeletronic chip devices which utilize controlled electrophoretic fields for multiplex DNA hybridization and other genomic applications. Electrophoresis 21:157-164.

5.Petrik, J. 2001. Microarray technology: the future of blood testing? Vox. Sang. 80:1-11.

6.Shi, M.M. 2001. Enabling large-scale pharmacogenetic studies by high-throughput mutation detection and genotyping technologies. Clin. Chem. 47:164-172.

7.Small, J., D.R. Call, F.J. Brockman, T.M. Straub, and D.P. Chandler. 2001. Direct detection of 16S rRNA in soil extracts by using oligonucleotide microarrays. Appl. Environ. Microbiol. 67:4708-4716.

8.Taniguchi, M., K. Miura, H. Iwao, and S. Yamanaka. 2001. Quantitative assessment of DNA microarrays - comparison with Northern blot analyses. Genomics 71:34-39.

9.Westin, L., C. Miller, D. Vollmer, D. Canter, R. Radtkey, M. Nerenberg, and J.P. O'Connell. 2001. Antimicrobial resistance and bacterial identification utilizing a microelectronic chip array. J. Clin. Microbiol. 39:1097-1104.

10.Zammatteo, N., L. Jeanmart, S. Hamels, S. Courtois, P. Louette, L. Hevesi, and J. Remacle. 2000. Comparison between different strategies of covalent attachment of DNA to glass surfaces to build DNA microarrays. Anal. Biochem. 280:143-150.

The authors wish to thank Elise Langdon-Neuner for assistance in editing the manuscript. Address correspondence to Dr. Klaus Zimmermann, Baxter BioScience, In- dustriestrasse 72, A-1221 Vienna, Austria. e-mail:zimmerk@baxter.com

Received 1 February 2002; accepted 30 April 2002.

Thomas Eiter, Klaus

Zimmermann, and Friedrich Scheiflinger

Baxter BioScience

Vienna, Austria

For reprints of this or any other article, contact Reprints@BioTechniques.com

\section{Internal Controls for Normalizing DNA Arrays}

BioTechniques 33:496-502 (September 2002)

General challenges of DNA array hybridization experiments are the specificity and reproducibility of these assays. We have previously addressed specificity through a combination of enzymatic labeling of DNA probes with the possibility of detecting several targets simultaneously by array hybridization (3-5). High signal-to-noise ratios, caused by the specificity in the enzymatic labeling reaction, were obtained (4). However, reproducibility is based on the signal intensities of the hybridization regions alone. Thus, experiment-to-experiment variation is dependent on the spot shape/size, amount of probe used, and the hybridization conditions. If the assay is not highly standardized, then a relatively high variance could be introduced into the results.

Here 5' Cy5-labeled internal control probes were included in the assay. The control probes were identical to the probes used in sequence-specific labeling. The signals from the sequence-specific labeled probes (TAMRA-ddCTP; Perkin Elmer Life Sciences, Boston, MA, USA) are measured relative to the internal control probes (Figure 1). The 